\section{Randomisierte Doppelblindstudie (third place blinded) zur Untersuchung der Wirksamkeit und Nebenwirkungen von Methacholin im unspezifischen bronchialen Provokationstest}

\begin{abstract}
Zusammenfassung: Für die Zulassung von Methacholin als Diagnostikum der unspezifischen bronchialen Hyperreaktivität (BHR) wurden die Unbedenklichkeit und Wirksamkeit in einer von dritter Stelle verblindeten Studie (third place blinded) überprüft. 61 Patienten mit anamnestischen Hinweisen auf eine BHR wurden in die Studie aufgenommen. Davon konnten in 56 Fällen komplette Daten erhalten werden. 27 Studienteilnehmer erhielten Verum (Methacholin 0,33\%) in einem 5-Stufen-Test mit der Reservoirbeutel-Methode; 29-mal wurde in gleicher Art Plazebo ( $\mathrm{NaCl}$ 0,9\%) appliziert. Dabei erfolgten Verdoppelungen der Dosen durch Vergrößerung des Aerosolvolumens. Zielparameter waren eine Verdoppelung des basalen spezifischen Atemwegswiderstandes (sRt), der gleichzeitig mindestens 2,0 $\mathrm{kPa}^{*} \mathrm{~s}$ erreichen mußte. In der Verumgruppe erreichten zehn Patienten (33,3\%) die Zielkriterien im Sinne einer positiven Test-Reaktion, während in der Plazebogruppe nur ein Patient reagierte (3,5\%). Es bestand ein signifikanter Zusammenhang zwischen der sRt-Änderung und der kumuliven MCH-Dosis $(p<0,002)$. In der Plazebo-Gruppe war eine solche Beziehung erwartungsgemäß nicht nachweisbar $(p=0,20)$. Nebenwirkungen traten nicht auf. Der 5-Stufen-Test mit 0,33\%igem Methacholin erwies sich zur Diagnostik der BHR als gut geeignet. Sein wesentlicher Vorteil ist die kurze Testzeit (ca. 20 min) und daß keine Verdünnungsreihen benötigt werden.
\end{abstract}

Randomised Double-Blind Study (Third Place Blinded) to Examine the Effectivity and Side Effects of Metacholine in the Non-Specific Bronchial Provocation Test: Softly and effectiveness of methacholine for the diagnosis of non-specific bronchial hyperresponsiveness [BHR] were tested in a third place blinded study. 61 patients suspected to suffer from BHR took part. 56 subjects provided complete data and were included in the study. 27 participants were challenged with methacholine $0.33 \%$ (verum) and 29 patients received $\mathrm{NaCl} 0.9 \%$ (placebo). The challenge was applied as 5 -step-test using a storage bag. The doses were elevated by doubling the aerosol volume. A positive test result was assumed when basal specific airway resistance (sRt) reduplicated and simultaneously $2.0(\mathrm{kPa} * \mathrm{~s})$ was attained. Ten out of 27 subjects in the verum group (33.3\%) had a positive test result whereas in the placebo group only one subject showed a reaction (3.5\%). A statistically significant association between the change of sRt and the cumulative methacholine dose was confirmed in the verum group $(p<0.002)$, whereas this effect could not be observed in patients challen-

Pneumologie 2000; 54: 99-103

(C) Georg Thieme Verlag Stuttgart · New York ISSN 0934-8387

\author{
H. Huber ${ }^{1}$, R. Lauschner ${ }^{2}$, F. Papenfuss ${ }^{1}$, H. Allmers ${ }^{1}$, \\ X. Baur ${ }^{1}$ \\ ${ }^{1}$ Berufsgenossenschaftliches Forschungsinstitut für Arbeitsmedi- \\ zin (BGFA), Institut an der Ruhr-Universität Bochum, \\ Direktor: Prof. Dr. med. Xaver Baur Bochum \\ 2 LINDOPHARM GmbH, Hilden
}

ged with placebo $(p=0.20)$. Side effects did not occur. We conclude that inhalative challenge with methacholine $0.33 \%$ applied as a 5-step-test is suitable to objectify BHR. The substantial benefit of the applied test scheme is the short range of time in which the challenge can be performed (approximately 20 $\mathrm{min}$ ) and that dilution series of the test substance (methacholine $0.33 \%$ ) are not required.

\section{Einleitung}

Die weltweit gebräuchlichste Substanz zur Objektivierung der bronchialen Hyperreaktivität ist Methacholin [23]. Die European Respiratory Society empfiehlt diese Substanz zur Durchführung des bronchialen Hyperreaktivitätstestes wegen seiner geringeren Nebenwirkungen [24]. In den USA ist Methacholin zur Diagnostik der bronchialen Hyperreaktivität von Patienten mit klinisch inapparenter Asthmasymptomatik von der FDA zugelassen.

Im deutschen Sprachraum wird dieses Pharmakon ebenfalls häufig angewandt, obwohl es ebenso wie auch andere pharmakodynamische Substanzen für der bronchiale Hyperreaktivitätstestungen vom Bundesinstitut für Arzneimittel und Medizinprodukte bisher noch nicht zugelassen ist. Der Arbeitskreis „Bronchiale Provokationstests“ der deutschen Gesellschaft für Pneumologie hat Leitlinien für die bronchiale Provokation erarbeitet und sich dabei auf die MethacholinApplikation mittels der Reservoirbeutelmethode in fünf Stufen (Einkonzentrationstest) bezogen [5].

Für die Zulassung des Diagnostikums sollte jetzt in einer von dritter Stelle verblindeten Studie (third place blinded) die Unbedenklichkeit und Wirksamkeit von Methacholin überprüft werden. Sämtliche Studienteilnehmern waren Gutachtenpatienten mit begründetem Verdacht auf das Vorliegen einer berufsbedingten obstruktiven Atemwegserkrankung (Berufskrankheiten-Nrn. 4301, 4302 oder 1315).

\section{Patienten und Methoden}

\section{Patienten}

Vom 2.2.1997 bis 16.10.1997 wurden nach Randomisierung der Studienmedikation insgesamt 61 konsekutive Patienten mit dem Verdacht auf eine berufsbedingte obstruktive Atemwegserkrankung konsekutiv in die Studie aufgenommen. Alle berichteten über wiederholte arbeitsplatzbezogene Atemnot- 
zustände beim Umgang mit allergisierenden, chemisch-irritativen oder inhalativ-toxischen Arbeitssubstanzen. Nach Ausschluß von Kontraindikationen $[5,12]$ erhielten die Studienteilnehmer entweder 0,33\%iges Methacholin (w/v) oder physiologische Kochsalzlösung im 5-Stufen-Test.

Von den 61 Probanden waren 35 (57\%) männlichen und 26 (43\%) weiblichen Geschlechts. Das mittlere Alter betrug 41 ( \pm 13 ; median 39 ) Jahre. Größe und Gewicht zeigten die typischen geschlechtsspezifischen Unterschiede, jedoch keine Auffälligkeiten.

Zwischen den Behandlungsgruppen (Verum- und Plazebogruppe) bestanden keine erkennbaren Unterschiede hinsichtlich Alter, Größe und Gewicht.

Die Studie wurde von der Ethik-Kommission der Ruhruniversität Bochum genehmigt. Die Studienteilnehmer wurden eingehend über die Untersuchung aufgeklärt. Nur nach schriftlicher Zustimmung erfolgte eine Testung.

\section{Methacholin-Provokationstest}

Sämtliche Provokationen (Studienmedikation und Plazebo) erfolgten mit dem Düsenvernebler Pari-Provokationstest II (Firma Medanz, Starnberg). Hierbei wird in einem bis maximal $10 \mathrm{~L}$ fassenden Reservoirbeutel mittels eines pressluftgetriebenen Düsenverneblers ein Aerosol der Provokationssubstanz erzeugt [15]. Die Verneblung mit dem Pari-Provokationstest II erfolgt zeitgesteuert. Nach dem Einstellen der gewünschten Aerosolmenge, welche der Reservoirbeutel auffängt, wird vor dem Inhalationsvorgang 1 min gewartet, damit größere Partikel an der Beutelwand sedimentieren können. Die Inhalation des Methacholinaerosols erfolgte in Form tiefer Inspirationen nahe der Vitalkapazität ( $\geq 5 \mathrm{~s}$ pro Atemzug), bis der Beutel leergeatmet war. Während der Inhalationen und den lungenfunktionsanalytischen Messungen trugen die Patienten eine Nasenklemme.

Unter Berücksichtigung der von Knoch et al. [14] ermittelten Leistung des Verneblers ergeben sich unter Verwendung einer 0,33\%igen Methacholin ${ }^{1}$-Lösung die nachfolgenden Dosierungen am Mundstück (Tab.1):

Tab. 1 Methacholindosen ${ }^{1}$ kumulativ berechnet nach Knoch et al. [14]

\begin{tabular}{|c|c|c|c|c|c|}
\hline Teststufe & 1 & 2 & 3 & 4 & 5 \\
\hline Verneblerlaufzeit (s) & 6 & 12 & 24 & 48 & 96 \\
\hline Aerosolvolumen (I) & 0,5 & 1 & 2 & 4 & 8 \\
\hline Einzeldosis $\mathrm{MCH}^{1}$ (mg) & 0,015 & 0,030 & 0,061 & 0,122 & 0,243 \\
\hline kumulative Dosis $\mathrm{MCH}^{1}(\mathrm{mg})$ & 0,015 & 0,045 & 0,106 & 0,228 & 0,471 \\
\hline
\end{tabular}

${ }^{1}$ Das verwendete Methacholin $0,33 \%$ ist der Provokit $^{\circledR}$ M 0,33\% der Firma Lindopharm GmbH, 40729 Hilden.

In der Plazebogruppe wurde analog unter Verwendung physiologischer Kochsalzlösung vorgegangen (Tab. 2):
Tab. 2 NaCl-Dosen nicht-kumulativ berechnet nach Knoch et al. [14]

\begin{tabular}{llllll}
\hline Teststufe & 1 & 2 & 3 & 4 & 5 \\
\hline Verneblerlaufzeit (s) & 6 & 12 & 24 & 48 & 96 \\
Aerosolvolumen (I) & 0,5 & 1 & 2 & 4 & 8 \\
Einzeldosis $\mathrm{NaCl}(\mathrm{mg})$ & 0,041 & 0,081 & 0,162 & 0,324 & 0,648
\end{tabular}

\section{Lungenfunktionsuntersuchungen}

Die Lungenfunktion wurde mit einem volumenkonstanten Bodyplethysmographen gemessen (MasterLab-Body, Firma Jaeger, Würzburg). Vor der ersten Applikation der Testsubstanz oder des Plazebos erfolgte eine lungenfunktionsanalytische Untersuchung. Nach jeder Provokationsstufe wurde bodyplethysmographisch die spezifische Resistance ermittelt, in einigen Fällen auch FVC und $\mathrm{FEV}_{1}$ [26]. Als Referenzwerte dienten die Sollwerte nach Quanjer et al. [22].

\section{Abbruchkriterien und Testende}

Zielparameter war die spezifische Resistance (sRt). Der Provokationstest war beendet, wenn mindestens eine Verdoppelung der basalen sRt und mindestens ein Wert von $2,0 \mathrm{kPa} \cdot \mathrm{s}$ erreicht wurde. Wenn die Meßwerte unter diesen Zielgrößen lagen, d.h. sich keine signifikante Atemwegsobstruktion einstellte, wurden alle fünf Dosen der Testsubstanz bzw. des Plazebos appliziert. Die Testreaktionen stuften wir als positiv ein, wenn eine signifikante bronchiale Reaktion bei einer kumulativen Dosis von $\leq 0,3 \mathrm{mg}$ Methacholin auftrat und sich die bronchiale Antwort somit von einem gesunden Kontrollkollektiv unterschied [12].

Bei jedem Probanden erfolgte unabhängig davon, ob eine signifikante Atemwegsobstruktion auftrat, nach der letzten Dosisstufe eine Bronchospasmolyse mittels eines Dosieraerosols ( $3 \times 0,1 \mathrm{mg}$ Salbutamol).

\section{Statistik}

Die statistische Auswertung der klinischen Studie erfolgte durch das Statistische Institut für Marketing und Wissenschaft (SIMW GmbH), Dr. Martin Eckert, 40667 Meerbusch.

Die ergänzenden Tabellen und Grafiken wurden mit der Tabellenkalkulation Microsoft ${ }^{\circledR}$ Excel $^{\circledR}$ erstellt und berechnet.

\section{Ergebnisse}

\section{Auswertbarer Datenumfang}

61 Patienten wurden in die Studie aufgenommen. Davon kamen 56 Patienten zur Auswertung. 27 Studienteilnehmer erhielten Verum (Methacholin 0,33\%), 29-mal wurde Plazebo ( $\mathrm{NaCl} \mathrm{0,9 \% )} \mathrm{verabreicht.}$

In fünf Fällen konnte das Protokoll nicht wie vorgesehen durchgeführt oder beendet werden. Dabei waren in drei Fällen bei der Basis-Lungenfunktionsuntersuchung Kontraindikationen zur Durchführung einer bronchialen Provokation festzustellen. In zwei Fällen zogen die Probanden ihr Ein- 
verständnis, an der Studie teilzunehmen, zurück bzw. war eine Auswertung der Messungen nicht möglich.

\section{Begleitende Untersuchungen}

Diese umfaßten Anamnese, Vitalparameter (Puls, Blutdruck, Ruhe-EKG), körperliche Untersuchung, laborchemische Untersuchungen (Blutbild, Retentionswerte, Elektrolyte, SerumElektrophorese, Transaminasen, $\gamma$-GT, LDH, CPK und Urinstatus) jeweils 24 Stunden vor und 24 Stunden nach Applikation der Testsubstanz.

In keinem Fall traten signifikante Veränderungen der untersuchten Parameter auf, welche auf die verabreichte Substanz zurückzuführen waren. Es zeigten sich auch keine signifikante Befunddifferenzen zwischen der Verum- und Plazebogruppe. Sehr schwere asthmatische Reaktionen waren ebenfalls nicht zu verzeichnen.

\section{Lungenfunktionsänderungen nach Verum- und Plazebogabe}

Die sRt-Mittelwerte in der Verum-Gruppe steigen mit zunehmender Methacholin-Dosis an, während in der PlazeboGruppe nur ein leichter Aufwärtstrend zu erkennen ist.

Vergleicht man die positiven Testergebnisse im Sinne der eingangs genannten Kriterien für den sRt, ist in der VerumGruppe in zehn von 27 Fällen (37\%) eine signifikant positive Testreaktion aufgetreten. In der Plazebo-Gruppe wurden bei Verabreichung aller fünf Teststufen die Zielkriterien (Verdoppelung der Baseline um mindestens $100 \%$ und auf einen Wert von $\geq 2 \mathrm{kPa} \cdot \mathrm{s}$ ) nur in einem von 29 Fällen erreicht (dabei fiel die $\mathrm{FEV}_{1}$ um $16 \% \mathrm{ab}$ ).

Im zweiseitigen Fisher's Exact-Test ergibt sich für die VerumGruppe im Vergleich zur Plazebo-Gruppe ein p-Wert von 0,00196 , welcher auf dem $1 \%$-Niveau signifikant ist.
Man erkennt einen signifikanten Zusammenhang zwischen der sRt-Änderung und der kumuliven MCH-Dosis $(\mathrm{p}<0,002)$. In der Plazebo-Gruppe ist eine solche Beziehung nicht nachweisbar ist ( $\mathrm{p}=0,20)$ (Tab. 3, Abb. 1 und 2).

\section{Diskussion}

Zur Erfassung der unspezifischen bronchialen Hyperreaktivität existieren mehrere Testschemata, die sich hinsichtlich der Applikation, Dosierung und der Reaktionserfassung unterscheiden $[4,6,10,12]$. Der von uns angewandte Test hat im deutschen Sprachraum eine breite Anwendung gefunden und wurde von der Deutschen Gesellschaft für Pneumologie empfohlen [5].

Die prinzipielle Eignung von Methacholin zur Erfassung der unspezifischen bronchialen Hyperreaktivität ist in der internationalen Literatur vielfach belegt $[1,7,8,13,17,18,24,25]$ und konnte auch in der jetzigen „third place blinded Doppelblindstudie“ gegenüber Plazebo nachgewiesen werden.

Sämtliche Patienten, die nicht in die Studie aufgenommen werden konnten (violaters), wiesen lungenfunktionsanalytische Kontraindikationen oder Symptome auf, welche unabhängig von der Studienmedikation waren. Unter Berücksichtigung der bekannten Kontraindikation [5,12] zeichnet sich Methacholin durch äußerst seltene ernsthafte unerwünschte Wirkungen aus. Auch in dieser Studie waren keine Nebenwirkungen festzustellen.

Hervorzuheben ist, daß alle Patienten, welche 0,33\%iges Methacholin erhielten, im Mittel und individuell nach Gabe einer üblichen Dosis eines Bronchospasmolytikums wieder Basalwerte der spezifischen Resistance zeigten (95\%-Konfidenzintervall: $-10,33 \%$ bis 16,60\% des Basalwertes). Diese Tatsache verdeutlicht unsere Erfahrung, daß auch starke asthmatische Reaktionen auf Methacholin therapeutisch gut beherrschbar sind.

\begin{tabular}{|c|c|c|c|c|c|c|c|}
\hline \multirow[t]{2}{*}{ Verum $(n=27)$} & \multicolumn{3}{|c|}{$\mathrm{sR}_{\mathrm{t}}$ absolut $\left[\mathrm{kPa} *^{*} \mathrm{~s}\right]$} & \multicolumn{4}{|c|}{$s R_{\mathrm{t}}$-Änderung [\%] } \\
\hline & Anzahl & Mittelw. & Median & Std.Abw. & Mittelw. & Median & Std.Abw. \\
\hline Baseline & 27 & 0,80 & 0,70 & 0,39 & $-1-$ & $-1-$ & $-1-$ \\
\hline Stufe $1(0,0152 \mathrm{mg} \mathrm{MCH})$ & 27 & 1,10 & 0,82 & 0,82 & 36,33 & 12,24 & 56,85 \\
\hline Stufe $2(0,0304 \mathrm{mg} \mathrm{MCH})$ & 26 & 1,16 & 0,86 & 0,85 & 41,42 & 31,31 & 47,43 \\
\hline Stufe $3(0,0607 \mathrm{mg} \mathrm{MCH})$ & 24 & 1,33 & 0,87 & 0,99 & 70,01 & 37,31 & 71,64 \\
\hline Stufe $4(0,1214 \mathrm{mg} \mathrm{MCH})$ & 21 & 1,27 & 0,85 & 0,98 & 76,67 & 48,10 & 70,19 \\
\hline Stufe 5 (0,2429 mg MCH) & 18 & 1,32 & 0,98 & 1,45 & 137,49 & 63,23 & 257,76 \\
\hline nach Br.spasmolyse & 27 & 0,75 & 0,65 & 0,27 & 3,16 & $-5,56$ & 34,10 \\
\hline \multirow[t]{2}{*}{ Plazebo $(n=29)$} & \multicolumn{3}{|c|}{$\mathrm{sR}_{\mathrm{t}}$ absolut $\left[\mathrm{kPa}^{*} \mathrm{~s}\right]$} & & \multicolumn{3}{|c|}{ sR $\mathrm{t}_{\mathrm{t}}$-̈̈nderung [\%] } \\
\hline & Anzahl & Mittelw. & Median & Std.Abw. & Mittelw. & Median & Std.Abw. \\
\hline Baseline & 29 & 0,95 & 0,90 & 0,25 & $-1-$ & $-1-$ & $-1-$ \\
\hline Stufe $1(0,0405 \mathrm{mg} \mathrm{NaCl})$ & 29 & 1,00 & 0,93 & 0,36 & 4,73 & 5,36 & 24,19 \\
\hline Stufe $2(0,0810 \mathrm{mg} \mathrm{NaCl})$ & 29 & 1,02 & 0,96 & 0,40 & 5,60 & 6,19 & 25,16 \\
\hline Stufe $3(0,1620 \mathrm{mg} \mathrm{NaCl})$ & 29 & 1,03 & 0,91 & 0,44 & 7,30 & 3,23 & 32,51 \\
\hline Stufe 4 (0,3240 mg NaCl) & 28 & 1,03 & 0,95 & 0,39 & 7,90 & 10,53 & 24,71 \\
\hline Stufe 5 (0,6480 mg NaCl) & 28 & 1,10 & 1,04 & 0,46 & 14,83 & 4,07 & 32,76 \\
\hline nach Br.spasmolyse & 29 & 0,75 & 0,74 & 0,32 & $-22,17$ & $-25,32$ & 23,66 \\
\hline
\end{tabular}

Tab. 3 Änderung der spezifischen Resistance $\left(\mathrm{sR}_{\mathrm{t}}\right)$ in der Verum-(Methacholin 0,33\%) und Plazebogruppe $(\mathrm{NaCl} 0,9 \%)$ 


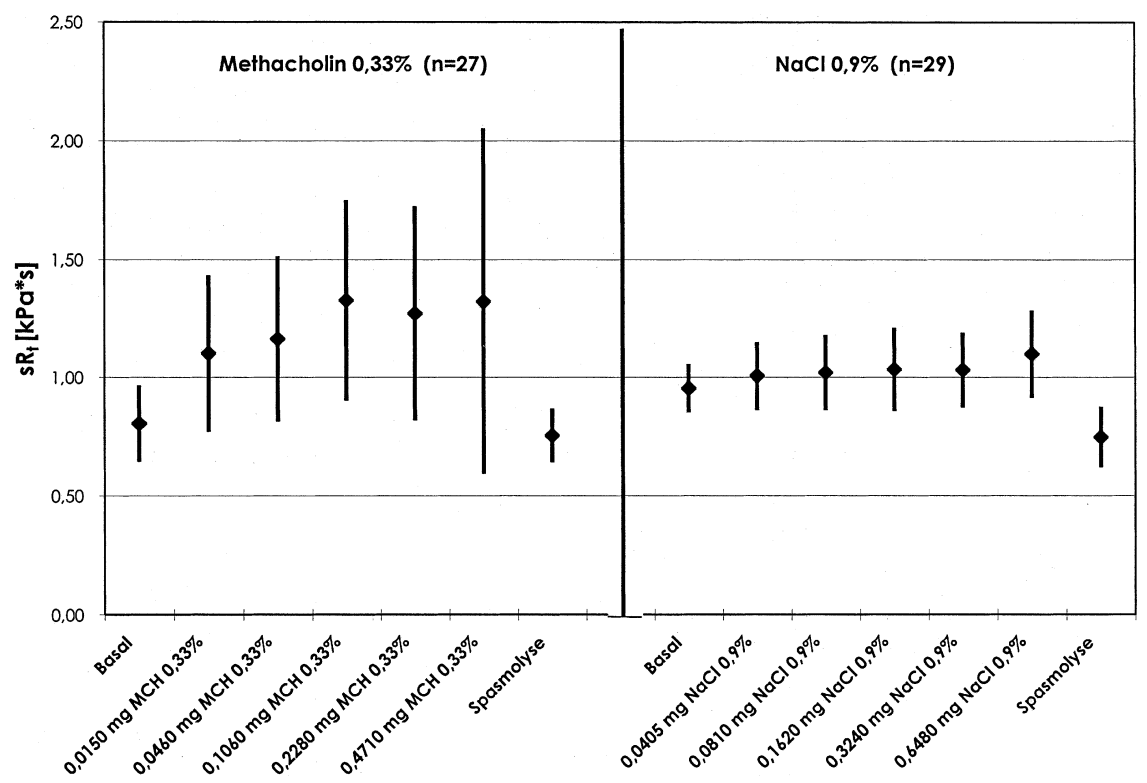

Abb. 1 Änderung der spezifischen Resistance in der Verum- (Methacholin 0,33\%) und Kontrollgruppe $(\mathrm{NaCl} 0,9 \%)$. Angegeben sind jeweils Mediane und Standardabweichungen.

Testergebnisse dieser Art hängen vom primären Zielparameter und somit von der Meßmethode und dem Patientengut ab. Im angloamerikanischen Sprachraum werden vielfach das Provokationsprotokoll nach Chai [4] oder dessen Modifikationen [24] angewandt. Hierbei wird Methacholin in Verdoppelungsdosen unter Verwendung aufsteigender Konzentrationen appliziert und als Zielkriterium für einen positiven Test eine $\mathrm{PD}_{20} \mathrm{FEV}_{1}$, d.h. ein $20 \%$ iger Abfall der FEV 1 bei einer kumulativen Methacholin-Dosis von meist 8 oder $12 \mathrm{mg}$ herangezogen.

In unserem Testschema wird nur eine 0,33\%ige MethacholinKonzentration benötigt und eine schrittweise, jeweils ca. 3fache Dosissteigerung durch eine leicht durchführbare Erhöhung der Aerosolmenge erzielt. Dies stellt eine erhebliche Vereinfachung für die medizinischen Hilfskräfte dar. Auch kann so der Provokationstest mit einem akzeptablen Zeitaufwand durchgeführt werden (ca. $20 \mathrm{~min}$ ). Nicht zuletzt ist eine erhöhte Anwendungssicherheit gegeben, weil die Verdünnungsreihen der Provokationssubstanz nicht mehr selbst hergestellt werden müssen.

Die im Vergleich zu den von Chai et al. [4] erheblich niedrigeren Provokationsdosen erklären sich im Wesentlichen dadurch, daß wir ein Aerosol mit einem viel kleineren Tröpfchendurchmesser einsetzten und daß nicht die vernebelte Methacholin-Menge, sondern die am Mundstück ankommende Methacholin-Menge (Knoch et al. [14]) als Berechnungsgröße berücksichtigt wird.

Die unspezifische bronchiale Hyperreaktivität, objektivierbar durch pharmakodynamischen Substanzen, ist unabhängig vom Testschema ein wesentliches Kennzeichen der obstruktiven Atemwegskrankheit und bleibt auch im krankheitsfreien Intervall nachweisbar. Darüber hinaus kann ein positives Testergebnis beispielsweise ein berufliches Risiko hinsichtlich

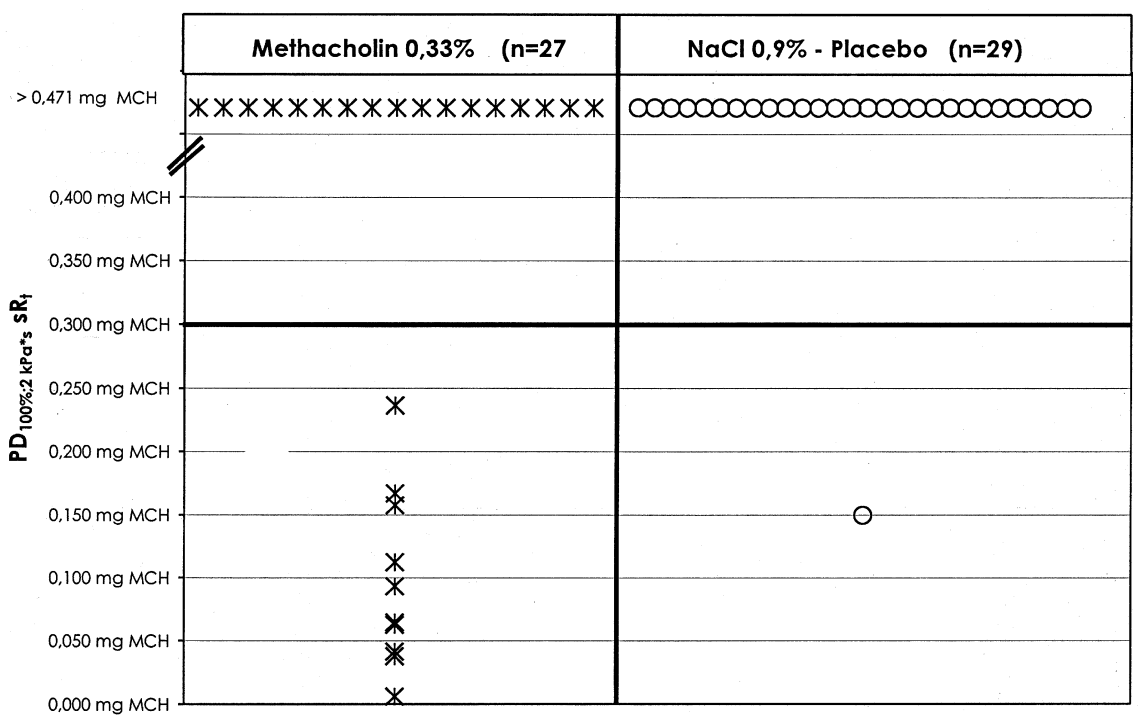

Abb. $2 \mathrm{PD}_{100 \% ; 2 \mathrm{kPa}^{*} \mathrm{~s}} s \mathrm{R}_{\mathrm{t}}$ aller ausgewerteten Studienteilnehmer. Die Verum-Ergebnisse (Methacholin 0,33\%) unterscheiden sich signifikant von jenen, welche mit Plazebo $(\mathrm{NaCl} 0,9 \%)$ erzielt wurden $[\mathrm{p}=0,00196$; zweiseitiger exakter Test von Fisher]. 
der Entwicklung einer Atemwegserkrankung aufdecken [3, 6, $9,11,16,19,20,21]$. Da der spezifische bronchiale Provokationstest mit Allergenen und insbesondere mit berufstypischen Irritantien aufwendig ist, wird oft mittels des unspezifischen Provokationstests eine diagnostische Aussage angestrebt. Bei unseren Patienten mit Verdacht auf eine berufsbedingte obstruktive Atemwegserkrankung zeigten 10 von 27 (37\%) der Untersuchten in der Verumgruppe eine bronchialobstruktive Reaktion auf Methacholin. Diese Anzahl entspricht unseren früheren Erfahrungen [2] und liegt deutlich über der Häufigkeit der bronchialen Hyperreaktivität in der Normalbevölkerung.

Andererseits ist bekannt, daß der spezifische Provokationstest nicht zu ersetzen ist und sich die Ergebnisse des Methacholin-Tests und des spezifischen Provokationstests erheblich voneinander unterscheiden können. Dieses trifft vor allem für Patienten mit Isocyanat-Asthma zu [2]. Abhängig vom untersuchten Kollektiv findet sich ein variabler Probandenanteil, welcher trotz gesicherter Asthmaerkrankung einen negativen Methacholin-Testbefund aufweist [2]. Hinsichtlich dieser Tatsache müssen weitere Untersuchungen zur Sensitivität des Methacholin-Tests durchgeführt werden. In diesem Zusammenhang ist von Interesse, inwieweit zusätzliche Kriterien, beispielsweise die standardisierte Anamnese, Atopiescores oder auch Belastungsuntersuchungen in der Diagnostik eines inapparenten Asthma bronchiale hilfreich sind.

Insgesamt kann die bronchokonstriktorische Wirksamkeit und Unbedenklichkeit von Methacholin zur Objektivierung der unspezifischen bronchialen Hyperreaktivität gezeigt werden.

\section{Literatur}

${ }^{1}$ Ammon J, Marek W, Baur X. Erstellung eines Normalkollektivs für den Methacholin-Provokationstest. Atemw Lungenkrkh 1993; 19: 303 - 304

2 Baur X, Huber H, Degens PO, Allmers H, Ammon J. Relation between occupational asthma case history, bronchial methacholine challenge, and specific challenge test in patients with suspected occupational asthma. Am J Ind Med 1998; 33 (2): $114-122$

${ }^{3}$ Britt EJ, Cohen B, Menkes H, Bleeker E. Airways reactivity and functional deterioration in relatives of COPD patients. Chest 1980; 77: 260 - 261

${ }^{4}$ Chai $\mathrm{H}$, Froelich LA et al. Standardisation of bronchial challenge procedures. J Allergy Clin Immunol 1975; 56: 323 - 327

${ }^{5}$ Klein Get al. Deutsche Gesellschaft für Pneumologie - Wissenschaftliche Arbeitsgruppe Leitlinien für die Durchführung bronchialer Provokationstests mit pharmakologischen Substanzen. Pneumologie 1998; 52 (4): $214-220$

${ }^{6}$ Eiser NM. Bronchial provocation tests. In: (Hrsg) Nadel JA, Pauwels R, Snashall PD. Bronchial hyperresponsiveness. Blackwell Oxford: 1987.173-254

${ }^{7}$ Enarson DA, Schulzer M, Vedal S, Chan-Yeung M. Summarizing methacholine bronchoprovocation data in epidemiological surveys. Bull Eur Physiopathol Resp 1987; 23: 387 - 389

${ }^{8}$ Goldstein MF, Pacana SM, Dvorin DJ, Dunsky EH. Retrospective analyses of methacholine inhalation challenges. Chest 1994; 105: $1082-1088$

${ }^{9}$ Hermann H. Bronchiale Hyperreaktivität und Krankheitsrisiko. Ergebnisse einer Longitudinal-Studie. Prax klin Pneumol 1983; 37: $670-672$
${ }^{10}$ Hoffarth HP, Reier W, Reichel G, Ulmer WT. Der inhalative 1Konzentrationstest zur Überprüfung der bronchialen Reagibilität. Pneumologie 1991; 45: 690 - 694

${ }^{11}$ Hopp R, Robert G, Townley G, Biven E, Bewtra AK, Nair NM. The presence of airway reactivity before development of asthma. Am Rev Respir Dis 1990; 141: 2 - 8

${ }^{12}$ Huber H, Ammon J, Baur X. Bronchialer Hyperreaktivitätstest (Kontraktionstest). In: Baur X (Hrsg.). Lungenfunktionsprüfung und Allergiediagnostik. München - Deisenhofen: Dustri-Verlag, 1998. $69-82$

${ }^{13}$ Huber H, Marek W, Baur X. Einfluß der arbeitsplatzbezogenen Isocyanat-Provokation auf die bronchiale Methacholin-Empfindlichkeit. Atemw Lungenkrkh 1994; 20: 661 (Abstract)

${ }^{14}$ Knoch M, Wunderlich E, Köhler D. Performance reproducibility of an aerosol delivery system for bronchial challenge tests. Abstract und Poster; Eur Resp 1995; J 8; (S 19): 104

${ }^{15}$ Köhler D, Schümichen C, Matthys H. Eine neue Apparatur zur interindividuellen dosisgenauen Applikation von Aerosolen. Klin Wschr 1985; 63 (IV): 232

${ }^{16}$ Muller BA, Leick CA, Suelzer M, Piyamahunt A, Richerson HB. Prognostic value of methacholine challenge in patients with respiratory symptoms. J Allergy Clin Immunol 1994; 94; 1: 78 87

${ }^{17}$ O'Connor GT, Sparrow D, Taylor D, Segal MR, Weiss ST. Analysis of dose-response curves to methacholine. An approach suitable for population studies. Am Rev Respir Dis 1987; 136: 1412 - 1417

18 O'Connor GT, Sparrow D, Weiss ST. Normal range of methacholine responsiveness in relation to prechallenge pulmonary function. The normative aging study. Chest 1994; 105: 661 - 666

${ }^{19}$ Orehek J, Gayrard P, Smith AP, Grimaud C, Charpin J. Airway response to carbachol in normal and asthmatic subjects. Distinction between bronchial sensitivity and reactivity. Am Rev Respir Dis 1977; 115: 937 - 943

${ }^{20}$ Orie NGM, Sluiter HJ, de Vries K, Tammeling GJ, Witkop J. The host factor in bronchitis. In: Orie NGM, Sluiter HJ (Hrsg.). Bronchitis. The Netherlands: Van Gorcum Press Assen, 1961. S43 - 59

${ }^{21}$ Piontek K, Hoffarth HP, Reichel G, Ulmer WT. Häufigkeit und Verlauf einer bronchialen Hyperreaktivität bei Bergleuten. Atemw Lungenkrkh 1990; 16: 261 - 263

${ }^{22}$ Quanjer PhH (Hrsg). Standardized lung function testing. Bull europ Physiopath resp 1983; 19 (S): 1 - 95

${ }^{23}$ Scott GC, Braun SR. A survey of the current use and methods of analysis of bronchoprovocation challenges. Chest 1991; 100: $322-328$

${ }^{24}$ Sterk PJ, Fabbri LM, Quanjer PhH, Cockcroft DW, O'Byrne PM, Anderson SD, Juniper EF, Malo JL. Airway responsiveness Standardized challenge testing with pharmacological, physiological and sensitizing stimuli in adults. Eur Respir J 1993; 6 (S): $53-83$

${ }^{25}$ Townley RG, Bewtra AK, Nair NM, Brodkey FD, Watt GD, Burke KM. Methacholine inhalation studies. J Allergy Clin Immunol 1979; 64; 8: 569 - 574

${ }^{26}$ Ulmer WT, Reichel G, Nolte D, Islam MS. Die Lungenfunktion. Stuttgart - New York: Georg Thieme Verlag, 1991

\section{Prof. Dr. med. X. Baur}

Berufsgenossenschaftliches Forschungsinstitut für Arbeitsmedizin (BGFA) Institut an der Ruhr-Universität Bochum Bürkle-de-la-Camp-Platz 1 44789 Bochum 